\title{
Valor pronóstico del nuevo sistema de etapificación internacional en mieloma múltiple. Comparación con el sistema de D urie-Salmon
}

\author{
Guillermo Conte $L^{1,7}$, Gastón Figueroa $M^{1}$, Vivianne Lois $V^{2}$, \\ María Elena Cabrera $\mathbf{C}^{3}$, Álvaro León $\mathbf{R}^{4}$, Hernán $\mathbf{G}$ arcía $\mathbf{L}^{5}$, \\ Hernán Rojas $\mathbf{R}^{6}$ \\ Prognostic value of the \\ new international staging system \\ in multiple myeloma. Comparison with \\ Durie-Salmon staging system
}

Background: Since 1975, the Durie-Salmon staging system (D\&S) has been a widely accepted prognostic classification of multiple myeloma (MM) patients. Recently, the new International Staging System (ISS) was developed using only the values of albumin and beta2-microglobulin. Aim: To compare survival of patients with MM treated in six medical centers in Chile according to the D\&S system and the new ISS. Material and methods: Retrospective analysis of demographic information, clinical features and survival rate of patients treated between 1998 and 2002, and grouped according to both systems. Results: Information of 81 patients aged 38 to 90 years (43 women) was retrieved. According D\&S system $11 \%$ were in stage I, $12 \%$ in stage II and $73 \%$ in stage III. According to ISS, $34 \%$ were in stage I, $35 \%$ in stage II and $31 \%$ in stage III. Median of survival of all patients was 32 months. Both staging systems had a prognostic value. However, median survival for the three stages of the ISS system was significantly different (67, 29 and 14 months in stages I, II and III, respectively, $\mathrm{p}=0.02$ ). Patients in advanced stages II and III of the ISS, had a higher frequency of anemia, hypercalcemia, renal failure and hypoalbuminemia. In stages II and III of ISS the presence of renal failure was associated with a non significantly different lower survival. Conclusions: The ISS is a simple and effective grouping method for patients with MM, that predicts survival. The presence of renal insufficiency might identify a subgroup of patients included in stages II and III of ISS with a higher mortality (Rev Méd Chile 2008; 136: 7-12).

(Key words: Kidney failure, chronic; Multiple myeloma; Neoplasm staging)

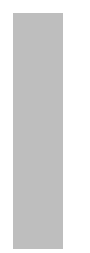

Recibido el 3 de mayo, 2007. Aceptado el 29 de agosto, 2007.

${ }^{1}$ Sección de Hematología, Hospital Clínico Universidad de Chile. ${ }^{2}$ Hospital Barros Luco T. ${ }^{3}$ Hospital Salvador. ${ }^{4}$ Universidad Austral, Hospital Regional de Valdivia. ${ }^{5}$ Hospital San Borja A. ${ }^{6}$ Hospital Sótero del Río. ${ }^{7}$ International Myeloma Foundation Latin America. Este trabajo fue financiado parcialmente por International Myeloma Foundation. Esta organización no tuvo participación en el diseño del estudio, en la recolección, análisis o interpretación de los datos, ni en la preparación y revisión del manuscrito.

Correspondencia a: Dr. Guillermo Conte L. Sección de

Hematología, Hospital Clínico Universidad de Chile. Santos

Dumont 999, Independencia, Santiago, Chile. Fax: 7777618.

E mail: gfconte@gmail.com 
$\mathrm{L}$ a sobrevida de los pacientes con Mieloma Múltiple (MM) es muy variable, con una mediana de 2,5 a 3 años ${ }^{1}$. Desde 1975 se ha utilizado la clasificación de Durie y Salmon (D\&S) la cual, para determinar el pronóstico de los pacientes con $\mathrm{MM}^{2}$, se basa en la masa tumoral estimada. Sin embargo, desde hace años se reconoce la necesidad de mejorar este sistema de etapificación ${ }^{3}$. Nuevos estudios y el avance tecnológico revelaron la existencia de nuevos parámetros de significación pronóstica: beta2-microglobulina, índices de actividad proliferativa de las células plasmáticas (Plasma cell labeling index y la proporción de células en fase S), deshidrogenasa láctica, proteína $\mathrm{C}$ reactiva, alteraciones citogenéticas y perfil de expresión génica ${ }^{4,5}$. Recientemente una serie de expertos internacionales, utilizando un registro de 10.750 pacientes, lograron desarrollar un nuevo sistema de clasificación (International Staging System, ISS) que permite obtener en forma fácil y precisa el pronóstico ${ }^{6}$.

El objetivo de este estudio es comparar la sobrevida de una serie de pacientes con MM tratados en 6 centros hospitalarios en Chile de acuerdo a la clasificación de Durie-Salmon y a la nueva clasificación internacional.

\section{MATERIAL Y MÉTODO}

Este es un estudio retrospectivo, multicéntrico, en el cual participaron 6 centros hospitalarios. Cinco hospitales correspondían a distintas áreas de Santiago: Hospital Salvador, Hospital Barros Luco, Hospital San Borja Arriarán, Hospital Clínico de la Universidad de Chile, Hospital Sótero del Río. El otro centro participante fue el Hospital de Valdivia, perteneciente a la Universidad Austral de Valdivia. Se utilizó una hoja de registro diseñada previamente, en la que cada centro recolectó, en forma retrospectiva, información clínica, de laboratorio y sobrevida de los pacientes diagnosticados entre los años 1988 a 2002. A cada centro se le solicitó específicamente registrar la etapa según D\&S y los niveles de albuminemia y beta2microglobulina que son los dos parámetros que utiliza el ISS para la etapificación a los pacientes en los siguientes 3 estadios:
Estadio I: beta2-microglobulina $<3,5 \mathrm{mg} / \mathrm{L}$ y albuminemia $\geq 3,5 \mathrm{~g} / \mathrm{dL}$. Estadio II: beta2-microglobulina $<3,5 \mathrm{mg} / \mathrm{L}$ pero albuminemia $<3,5 \mathrm{~g} / \mathrm{dL}$; beta2-microglobulina entre 3,5 a $5,4 \mathrm{mg} / 1$ independiente de albuminemia. Estadio III: beta2microglobulina $\geq 5,5 \mathrm{mg} / \mathrm{L}$.

Para comparar los distintos grupos se calculó la mediana de sobrevida (en meses) y se generaron curvas de sobrevida con el método de Kaplan Meier. Las variables categoriales y continuas se compararon con las pruebas de Chi-cuadrado y la prueba no paramétrica de Mann-Whitney, tomando un $\mathrm{p}<0,05$ como estadísticamente significativo. Las pruebas estadísticas y curvas de sobrevida se realizaron con el programa estadístico SPSS 13.0 (SPSS Inc. Chicago, Illinois, USA).

\section{Resultados}

De un registro total de 245 pacientes, en 81 de ellos se obtuvo información sobre estadio según D\&S e ISS, debido a que la medición de beta2microglobulina plasmática no se encontraba disponible en todos los centros. La mediana de edad de estos 81 pacientes fue 65 años, con una relación de hombre y mujeres de 1:1,1. Las características demográficas y clínicas de los pacientes al momento del diagnóstico de MM se presentan en la Tabla 1.

La distribución de los pacientes según D\&S y el ISS fue diferente (Figura 1). En el primero $76 \%$ de los pacientes se encontraban en etapa III, a diferencia del ISS que distribuyó a los pacientes en 3 grupos de tamaño similar.

La mediana de sobrevida del total de pacientes fue de 32 meses. Las curvas de sobrevida de los pacientes agrupados según el sistema de D\&S y el ISS se presentan en la Figura 2 y las medianas de sobrevida respectivas se presentan en la Tabla 2. Aunque ambos sistemas entregan información pronóstica, el ISS separa a los pacientes en 3 grupos de sobrevida diferente (mediana 67 meses vs 29 meses vs 14 meses), alcanzando una diferencia estadísticamente significativa (Log Rank test $\mathrm{p}=0,016$ ).

Al comparar las características clínicas de los pacientes agrupados según el ISS (Tabla 3) encontramos que los estadios avanzados (II y III) se asocian a mayor frecuencia de anemia, hipercalce- 
Tabla 1. Características demográficas y clínicas de los casos de mieloma múltiple

\begin{tabular}{|lcc|}
\hline & $\mathrm{n}$ & $\%$ \\
\hline Total de pacientes & 81 & 100 \\
Hombre/mujer & $38 / 43$ & \\
Edad (años) mediana (rango) & $65(38-90)$ & 53,1 \\
Anemia $(\mathrm{Hb}<10 \mathrm{~g} / \mathrm{dL})$ & 43 & 20,9 \\
Hipercalcemia $(>10,5 \mathrm{mg} / \mathrm{dL})$ & 17 & 25,9 \\
Insuficiencia renal $(\mathrm{creat}>2 \mathrm{mg} / \mathrm{dL})$ & 21 & 11,1 \\
En programa de diálisis & 9 & 11,1 \\
Trombocitopenia $(<100.000 / \mu \mathrm{L})$ & 9 & 59,3 \\
Tipos de inmunoglobulinas & & 29,6 \\
$\quad$ IgG & 48 & 11,1 \\
$\quad$ IgA & 24 & \\
\hline
\end{tabular}

Creat: Creatininemia.

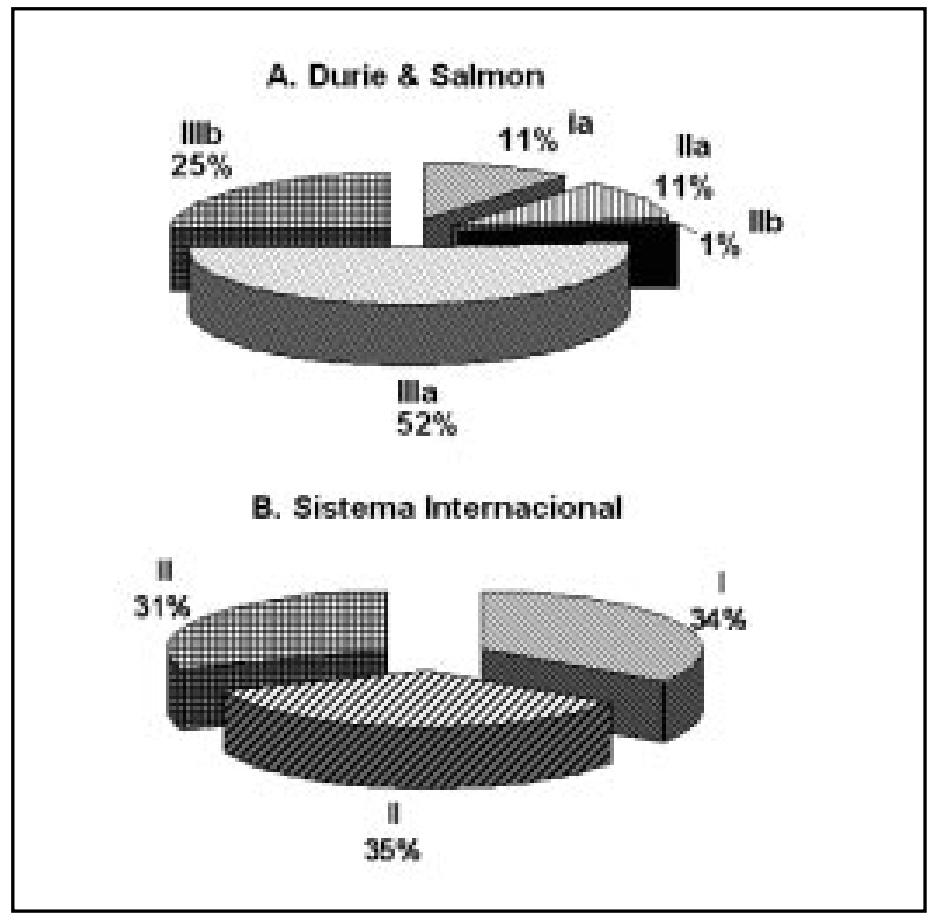

Figura 1. Distribución de los pacientes con MM según Durie y Salmon (A) y sistema internacional (B).

mia, insuficiencia renal e hipoalbuminemia. Destaca que 13 de los 21 pacientes con insuficiencia renal (creatininemia $>2 \mathrm{mg} / \mathrm{dL}$ ) se agrupan en la etapa III del ISS, representando la mitad (52\%) de los pacientes en esta etapa. Los pacientes en etapa
II y III del ISS con insuficiencia renal presentaron una mediana de sobrevida menor que los pacientes sin insuficiencia renal (II: 6 vs 29 meses; III: 8 vs 18 meses) aunque estas diferencia no fueron estadísticamente significativas. 


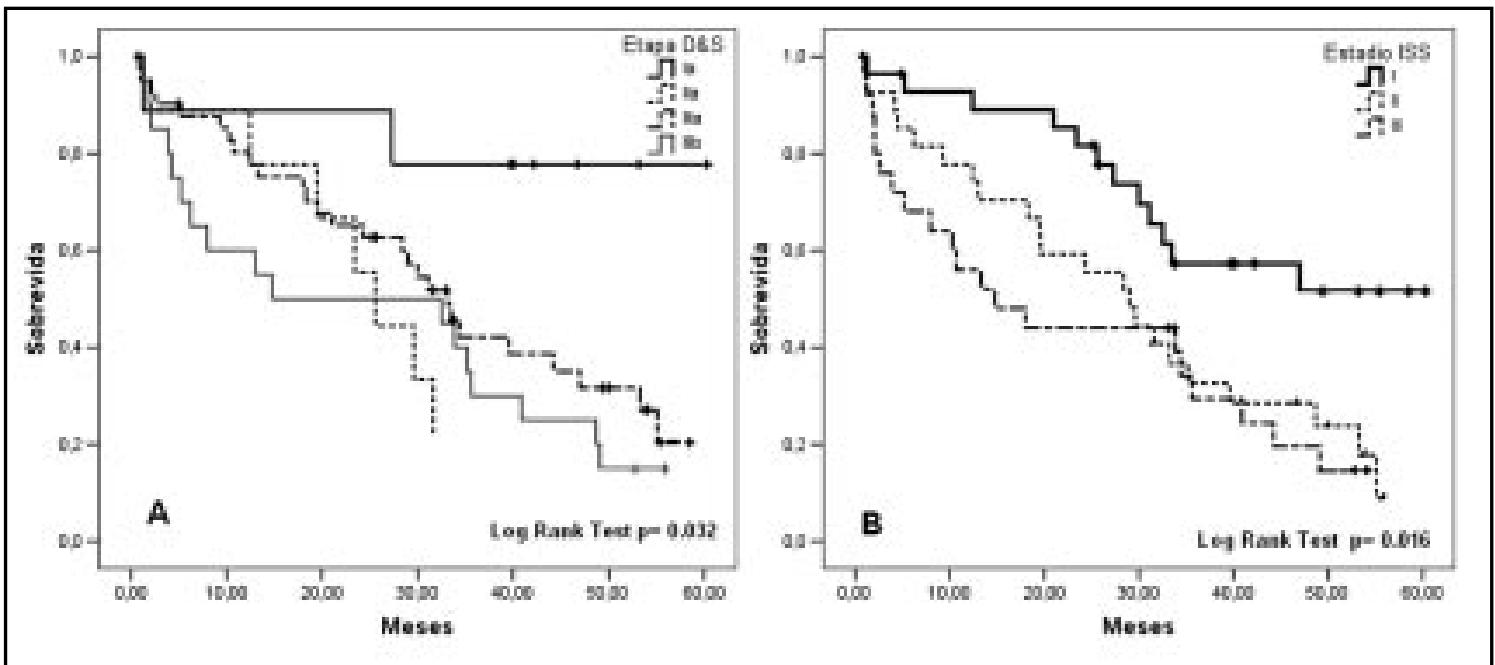

Figura 2. Sobrevida de los pacientes con MM según estadio de Durie y Salmon (A) y sistema internacional (B).

Tabla 2. Sobrevida según estadios de D urie-Salmon y el sistema internacional

\begin{tabular}{|cccccc|}
\hline Durie-Salmon & $\mathrm{n}$ & $\begin{array}{c}\text { Sobrevida mediana } \\
\text { (meses) }\end{array}$ & $\begin{array}{c}\text { Sistema } \\
\text { internacional }\end{array}$ & $\mathrm{n}$ & $\begin{array}{c}\text { Sobrevida mediana } \\
\text { (meses) }\end{array}$ \\
\hline IA & 9 & 67 & I & 28 & 67 \\
IIA & 9 & 26 & II & 28 & 29 \\
IIB & 1 & nd & III & 25 & 14 \\
IIIA & 42 & 33 & & & \\
IIIB & 20 & 15 & & & \\
\hline
\end{tabular}

nd: no determinado.

DisCUSIÓN

Hasta hoy el sistema de D\&S ha sido el más utilizado para la clasificación pronóstica de los pacientes con MM. Desde su publicación en 1975 el principal avance en la evaluación pronóstica de los pacientes con MM ha sido la incorporación de los valores de beta2-microglobulina. Sólo muy recientemente, el creciente conocimiento en la biología y genética del MM ha permitido determinar con mayor exactitud el pronóstico de los pacientes con esta neoplasia 4,5 .

Con el fin de obtener un mejor sistema de evaluación pronóstica, el nuevo ISS fue desarrollado con complejas técnicas de análisis univariable y multivariable en una población de 10.750 pacientes con MM no tratados previamente. Este estudio reveló que los niveles de albuminemia y beta2microglobulina son importantes factores de riesgo independientes de mortalidad con los cuales se pudo construir un simple sistema de etapificación ${ }^{6}$.

Desde hace años se conoce el valor pronóstico de los niveles de albuminemia ${ }^{7}$ y beta2-microglobulina en los pacientes con $\mathrm{MM}^{8,9}$, pero sólo recientemente se han comenzado a dilucidar los mecanismos subyacentes.

La albúmina es la proteína más abundante en el plasma y tiene importantes funciones biológicas $^{10}$. Los pacientes con cáncer y otros trastornos crónicos con frecuencia presentan hipoalbumine- 
Tabla 3. C aracterísticas demográficas y clínicas de los casos de mieloma múltiple agrupados de acuerdo al sistema internacional

\begin{tabular}{|c|c|c|c|c|}
\hline & \multicolumn{4}{|c|}{ Estadio SI } \\
\hline & I & II & III & $\mathrm{p}$ \\
\hline Total de pacientes & 28 & 28 & 25 & \\
\hline Hombre/mujer (n) & $12 / 16$ & $13 / 15$ & $13 / 12$ & ns \\
\hline Edad (años) (mediana) & 66 & 66 & 64 & ns \\
\hline Tipo cadena IgG/IgA/otra & $14 / 9 / 5$ & $17 / 8 / 3$ & 17/7/1 & ns \\
\hline$\%$ plasmocitosis medular & $35 \%$ & $51 \%$ & $50 \%$ & ns \\
\hline Lesiones líticas múltiples & $14(50 \%)$ & $14(50 \%)$ & $12(48 \%)$ & ns \\
\hline Trombocitopenia $(<100.000 / \mu \mathrm{L})$ & $3(11 \%)$ & $3(11 \%)$ & $3(12 \%)$ & ns \\
\hline Anemia $(\mathrm{Hb}<10 \mathrm{~g} / \mathrm{dL})$ & $7(25 \%)$ & $16(57 \%)$ & $20(80 \%)$ & $<0,001$ \\
\hline Hipercalcemia $(>10,5 \mathrm{mg} / \mathrm{dL})$ & 0 & $6(23 \%)$ & $11(46 \%)$ & $<0,001$ \\
\hline Insuficiencia renal (creat $>2 \mathrm{mg} / \mathrm{dL}$ ) & 0 & $8(28 \%)$ & $13(52 \%)$ & $<0,001$ \\
\hline En programa de diálisis & 0 & $4(14 \%)$ & $5(20 \%)$ & $<0,001$ \\
\hline Albuminemia ( $\mathrm{g} / \mathrm{dL}$-mediana) & 4,05 & 3,4 & 3,53 & $<0,001$ \\
\hline \multicolumn{5}{|l|}{ Beta2-microglobulina (mg/L) } \\
\hline Sin insuf. renal (mediana) & 1,9 & 3,9 & 9,0 & $<0,001$ \\
\hline Con insuf. renal (mediana) & & 1,3 & 16,6 & $<0,001$ \\
\hline
\end{tabular}

Las variables se expresan como mediana o número (porcentaje) según corresponda. ns: no significativo.

mia, la que se ha relacionado con un efecto inhibitorio de la síntesis hepática de albúmina mediada por citoquinas, principalmente TNF- $\alpha$ e IL- $6^{11,12}$. Estas dos citoquinas juegan importantes roles en la biología del MM. La IL-6 es secretada por las células estromales de la médula ósea en respuesta a la adhesión de células de mieloma o citoquinas (TNF- $\alpha$, VEGF, IL-1ß) secretadas por estas células y tiene importantes efectos en la proliferación y sobrevida de las células de mielo$\mathrm{ma}^{13}$. En pacientes con MM se encuentran niveles plasmáticos elevados de IL-6, que se han correlacionado con etapas avanzadas de la enfermedad, la respuesta al tratamiento y la sobrevida ${ }^{14,15}$. Estos hallazgos sugieren que en los pacientes con MM la hipoalbuminemia podría ser un marcador biológico de la actividad de estas dos citoquinas (TNF- $\alpha$, IL-6) explicando así su valor pronóstico.

La beta2-microglobulina es un polipéptido de bajo peso molecular sintetizado por todas las células nucleadas del organismo y forma la cadena liviana del complejo mayor de histocompatibilidad (HLA-clase I), cumpliendo un rol importante en el proceso de reconocimiento celular ${ }^{16}$. Es filtrada por el riñón y reabsorbida por el túbulo contorneado proximal. El incremento de los niveles plasmáticos de beta2-microglobulina puede deberse a disminución de la filtración glomerular o por aumento de la síntesis, lo que ocurre en patologías en las que el sistema inmunológico está involucrado como lupus eritematoso sistémico, artritis reumatoidea, mieloma múltiple, linfoma de células B, otras neoplasias y en algunas infecciones virales. En el caso de los pacientes con MM, los niveles de beta2-microglobulina se relacionan con la masa tumoral y la función renal ${ }^{8,9}$.

El presente estudio revela que el ISS es un método simple y efectivo de agrupar a los pacientes con MM en tres grupos de similar tamaño pero de pronóstico distinto (Figura 1, Tabla 2). Esta distribución es similar a la encontrada en el estudio original ${ }^{6}$, lo que apoya la validez externa del sistema. El principal defecto del sistema de D\&S es que agrupa a la mayoría de los pacientes (77\%) en etapa III, que corresponde a un grupo heterogéneo de pacientes con variable sobrevida.

Analizando la Tabla 2 podemos apreciar que el ISS logra concentrar en la etapa III pacientes de 
peor pronóstico, la mitad de los cuales (52\%) tienen insuficiencia renal, frecuencia similar a la encontrada en el estudio original $(43 \%)^{6}$. Dada la relación entre función renal y niveles de beta2microglobulina es difícil discriminar el valor pronóstico entre estos dos factores. Analizando por separado la sobrevida de los pacientes con y sin insuficiencia renal, los resultados sugieren que la presencia de insuficiencia renal podría identificar un subgrupo de pacientes dentro de las etapas II y III del ISS con una muy elevada mortalidad, lo que es semejante al valor pronóstico de la falla

\section{REFERENCIAS}

1. Dispenzieri A, Lacy M, Greipp P. Multiple Myeloma. En: Greer JP, Foerster J, Lukens JN, Rodgers GM, Paraskevas F, Glader BE (eds). Wintrobe's Clinical Hematology, $11^{\text {th }}$ Edition. Lippincot Williams and Wilkins, 2004; 2583-636.

2. Durie BGM, Salmon SE. A clinical staging system for multiple myeloma: Correlation of measured myeloma cell mass with presenting clinical features, response to treatment, and survival. Cancer 1975; 36: 842-54.

3. KYle RA. Why better prognostic factors for multiple myeloma are needed. Blood 1994; 83: 1713-6.

4. San Miguel JF, García-Sanz R. Prognostic features of multiple myeloma. Best Pract Res Clin Haematol 2005; 18: 69-83.

5. Stewart AK, Fonseca R. Prognostic and therapeutic significance of myeloma genetics and gene expression profiling. J Clin Oncol 2005 23: 6339-44.

6. Greipp PR, San Miguel J, Durie BG, Crowley JJ, Barlogie B, Bladé J et al. International staging system for multiple myeloma. J Clin Oncol 2005; 23: 3412-20.

7. Chen YH, Magalhaes MC. Hypoalbuminemia in patients with multiple myeloma. Arch Intern Med 1990; 150: 605-10.

8. Bataille R, Durie BGM, Grenier J. Serum beta 2 microglobulin and survival duration in multiple myeloma: A simple reliable marker for staging. $\mathrm{Br}$ J Haematol 1983; 55: 439-47.

9. Durie BGM, Stock-Novack D, Salmon SE, Finley P, Beckord J, Crowley J et al. Prognostic value of renal en el sistema de D\&S (ver Tabla 2). El valor pronóstico agregado de la presencia de insuficiencia renal en la sobrevida de los pacientes con MM en etapas II y III del ISS no fue analizado en el estudio original ${ }^{6}$.

El nuevo ISS es un aporte para la etapificación de los pacientes con MM, por su reproducibilidad y mayor poder pronóstico. Al permitir una estratificación pronóstica más adecuada de los pacientes, el ISS podría guiar de mejor forma el tratamiento de los pacientes con MM, el cual ha tenido importantes avances en los últimos años ${ }^{17}$.

pretreatment serum beta-2 microglobulin in myeloma: A Southwest Oncology Group Study. Blood 1990; 75: 823-30.

10. Quinlan GJ, Martin GS, Evans TW. Albumin: biochemical properties and therapeutic potential. Hepatology 2005; 41: 1211-9.

11. CHоJкіеR M. Inhibition of albumin synthesis in chronic diseases: molecular mechanisms. J Clin Gastroenterol 2005; 39 (4 Suppl 2): S143-S146.

12. Bologa RM, Levine DM, Parker TS, Cheigh JS, Serur D, Stenzel KH et al. Interleukin-6 predicts hypoalbuminemia, hypocholesterolemia, and mortality in hemodialysis patients. Am J Kidney Dis 1998; 32: 107-14.

13. Hideshima T, Bergsagel PL, Kuehl WM, Anderson KC. Advances in biology of multiple myeloma: clinical applications. Blood 2004; 104: 607-18.

14. LAUTA VM. Interleukin-6 and the network of several cytokines in multiple myeloma: an overview of clinical and experimental data. Cytokine 2001; 16: 79-86.

15. Thompson MA, Witzig TE, Kumar S, Timm MM, Haug J, Fonseca R et al. Plasma levels of tumour necrosis factor alpha and interleukin-6 predict progression-free survival following thalidomide therapy in patients with previously untreated multiple myeloma. Br J Haematol 2003; 123: 305-8.

16. Klein J, Sato A. The Hla System- First of Two Parts. N Engl J Med 2000; 343: 702-9.

17. Anderson KC, Pazdurn R, Farrell AT. Development of effective new treatments for Multiple Myeloma. J Clin Oncol 2005; 23: 7207-11. 